\title{
Tunnel-mediated coupling between antiferromagnetic thin films
}

\author{
Marie-Alix Leroy, ${ }^{1,2}$ Alexandre M. Bataille, ${ }^{1, *}$ Brahim Dkhil, ${ }^{3}$ Florence Porcher, ${ }^{1}$ Antoine Barbier, ${ }^{4}$ Vincent L. R. Jacques, \\ Yuan Lu, ${ }^{2}$ Christine Bellouard, ${ }^{2}$ Thomas Hauet, ${ }^{2}$ Sylvain Ravy, ${ }^{6}$ Javier Herrero-Martin, ${ }^{7}$ Christophe Gatel, ${ }^{8}$ \\ Karim Bouzehouane, ${ }^{9}$ Arsen Gukasov, ${ }^{1}$ Stéphane Andrieu, ${ }^{2}$ and Coriolan Tiusan ${ }^{2}$ \\ ${ }^{1}$ Laboratoire Léon Brillouin (UMR 012 CEA/CNRS), IRAMIS, CEA Saclay, bâtiment 563, 91191 Gif sur Yvette, France \\ ${ }^{2}$ Institut Jean Lamour (UMR 7198), Université de Lorraine, BP 239, 54500 Vandœuvre-lès-Nancy, France \\ ${ }^{3}$ Laboratoire Structure, Propriétés et Modélisation des Solides, UMR CNRS 8580, Ecole Centrale Paris, \\ Grande Voie des Vignes, 92295 Chatenay-Malabry Cedex, France \\ ${ }^{4}$ Service de Physique et Chimie des Surfaces et des Interfaces, IRAMIS, CEA Saclay, bâtiment 462, 91191 Gif sur Yvette, France \\ ${ }^{5}$ Laboratoire de Physique des Solides, Univ. Paris-Sud, CNRS, UMR 8502, F-91405 Orsay Cedex, France \\ ${ }^{6}$ Synchrotron SOLEIL, L'Orme des merisiers, Saint-Aubin BP48, 91192 Gif sur Yvette cedex, France \\ ${ }^{7}$ ESRF, Grenoble France \\ ${ }^{8}$ CEMES-CNRS, 29 rue Jeanne Marvig, 31055 Toulouse Cedex, France \\ ${ }^{9}$ Unité Mixte de Physique CNRS/Thales, 91767 Palaiseau, France and University of Paris-Sud, 91405 Orsay, France
}

(Received 24 July 2013; revised manuscript received 28 May 2014; published 22 July 2014)

\begin{abstract}
The study, tailoring, and control of the coupling between magnetic layers triggered the development of spintronics and has been up to now focused on systems comprising at least one ferromagnetic layer. Here we present a system where two antiferromagnetic layers separated by an ultrathin tunnel barrier interact with each other. We have studied with neutron and x-ray diffraction two sets of $\mathrm{Cr} / \mathrm{MgO} / \mathrm{Cr}$ trilayers, with either thin (five monolayers or less) or thick (eight monolayers or more) $\mathrm{MgO}$ layers, along with isolated $\mathrm{Cr}$ layers used as references. While the behavior of the trilayers with thick $\mathrm{MgO}$ layers can be explained by the strain state of each layer, that of trilayers with thin barriers cannot be related to the well known behavior of isolated chromium layers. We indeed report in these samples the presence of anomalous magnetic phases (modified propagation vector, direction of spins and propagation vectors inconsistent with the strain state of the sample) that we ascribe to the existence of a tunnel magnetic coupling between the AF layers through the insulating barrier. This unexpected finding opens opportunities for spintronics using solely antiferromagnets.
\end{abstract}

DOI: 10.1103/PhysRevB.90.035432

PACS number(s): 75.30.Fv, 75.70.Cn, 73.40.Gk

Antiferromagnetic (AF) thin films, made of materials which exhibit magnetic ordering but no net magnetic moment, are now widely used in spintronic devices, in which they play a secondary role by altering the coercivity of ferromagnetic layers through exchange bias. Yet in very recent reports, antiferromagnets are cast in a more active role. Indeed, both theoretical [1,2] and experimental [3] studies suggest that spin torque also exists within AF layers, with a much smaller critical current than in ferromagnetic layers. Furthermore, it has been recently shown that the switching of magnetic ordering parameter could be achieved using a magnetic field pulse [4] in a much more efficient way than for a ferromagnet. Within this quest to unravel the properties of AF thin films that are relevant for these uses, we performed a thorough study of the magnetic and structural properties of $\mathrm{Cr} / \mathrm{MgO} / \mathrm{Cr}$ trilayers. We show here that two antiferromagnets can be coupled across an ultrathin tunnel barrier, which opens original routes to design spintronics devices.

The discovery of giant magnetoresistance in $\mathrm{Fe} / \mathrm{Cr}$ superlattices [5] triggered an intense research effort on the magnetic properties of $\mathrm{Cr}$ thin films [6,7] and multilayers [8-12]. In all these examples, the magnetism remains purely itinerant since all layers are metallic. Here we chose to study the case of $\mathrm{Cr}$ layers separated by an insulating spacer. In this system, we expect to observe tunnel-mediated effects, particularly if the

*alexandre.bataille@cea.fr spacer layer is ultrathin so that electrons can tunnel between the two Cr electrodes.

$\mathrm{Cr}$ is one of the archetypal itinerant antiferromagnets. The peculiar topology of its Fermi surface [13] leads to nesting between electrons and holes pockets, which is the origin of the spin density wave (SDW) phase observed below the ordering temperature of $311 \mathrm{~K}$. The observation of such a complex behavior for a structurally simple compound ( $\mathrm{Cr}$ has a body centered cubic unit cell) makes chromium a much studied model system, used to shed light on complex phenomena such as quantum critical points $[14,15]$ or antiferromagnetic domain wall dynamics $[16,17]$.

The SDW is described by two vectors: The propagation vector (noted $\vec{Q}$ ) gives the direction and the period of the magnetic modulation, and the polarization vector (noted $\vec{S}$ ) gives the direction of the spins. Both vectors lie along the cube axes of the $\mathrm{Cr}$ unit cell. The incommensurability of the modulation is measured by the $\delta$ parameter: The length of the propagation vector can be written as $Q=1-\delta$ in reciprocal lattice units. The period of the SDW is then $a / \delta$, where $a$ is the lattice parameter of Cr. Along with the incommensurate SDW, a charge density wave (CDW) is also observed, with a propagation vector $2 \vec{Q}$. Depending on the relative orientation of $\vec{Q}$ and $\vec{S}$, the SDW is either longitudinal $(\vec{S} \| \vec{Q})$ or transverse $(\vec{S} \perp \vec{Q})$. A commensurate phase can also be observed in strained, defects-rich, or doped samples [13]. This large sensitivity of the SDW to strain and/or defects is both challenging and appealing: It requires a detailed structural characterization of the heterostructures yet at the 
same time it provides a very sensitive probe of the $\mathrm{Cr}$ electronic structure.

Using state of the art experimental tools including high resolution transmission electron microscopy, high resolution $\mathrm{x}$-ray diffraction, and neutron diffraction, we compared two sets of $\mathrm{Cr} / \mathrm{MgO} / \mathrm{Cr}$ trilayers with either thin [4-5 monolayers (ML)] or thick (8-11 ML) insulating barriers. While the behavior of the trilayers comprising thick $\mathrm{MgO}$ barriers are those expected from the structural properties of the individual $\mathrm{Cr}$ layer, the magnetic properties of the two $\mathrm{Cr}$ layers are no longer independent when the $\mathrm{MgO}$ thickness is $5 \mathrm{ML}$ or less. In particular, we observe anomalous incommensurate phases with an enlarged period, which evidences a strong alteration of the $\mathrm{Cr}$ electronic structure in these samples. These peculiarities cannot be explained by strain, finite size effects, or the presence of defects. We ascribe these peculiarities to a form of tunnel coupling between the two $\mathrm{Cr}$ layers across the ultrathin $\mathrm{MgO}$ barrier, making tunnel coupling a general feature of tunnel junctions including magnetically ordered electrodes [18].

The paper is organized as follows: We first describe the experimental methods used in this study in Sec. I, before presenting our experimental results in Sec. II. In particular, we provide the relevant information on the growth process and on the strain state of the samples (Sec. II A), and give our observation on the spin and charge density waves observed in our samples (Secs. II B and II C, respectively). We then discuss our results in Sec. III, first by excluding strain as the origin of the anomalous phases we observe (Sec. III A), and then by proposing a mechanism for the coupling we evidence, based on Cr electronic structure (Sec. III B).

\section{EXPERIMENTAL METHODS}

Sample were grown in a MBE setup, which is described in details elsewhere [18]. The base pressure of $\sim 10^{-10}$ Torr was reached thanks to the use of liquid $N_{2}$ cooled cryopanels. Growth was monitored in situ with a RHEED setup (the incident beam had a $30 \mathrm{keV}$ incident energy, and made a $4^{\circ}$ angle with the sample surface).

HRTEM observations were performed at $200 \mathrm{kV}$ using a FEI Tecnaï microscope equipped with a field emission gun and a Cs corrector which avoids delocalization effects at interfaces. The point resolution is $0.12 \mathrm{~nm}$. Thin samples were prepared in cross-sectional geometry using the usual mechanical polishing and a precision ion polishing system to achieve the electron transparency.

All samples were extensively studied using x-ray diffraction (XRD). Room temperature measurements were carried out at IJL using a PANalytical XPert Pro setup specially suited for the studies on thin films. The diffractometer was set in the socalled "triple axis" configuration, using $\mathrm{Cu} K_{\alpha_{1}}$ radiation $(\lambda=$ $1.54056 \AA$ ). Low temperature measurements were carried out with a homemade high resolution two circles diffractometer at SPMS, using a rotating anode $\mathrm{x}$-ray source, and a $\mathrm{Ge}$ (220) analyzer to select the $\mathrm{Cu} K_{\alpha_{1}}$ radiation. The extremely weak CDW satellite peaks were studied using the six circles diffractometers of the CRISTAL (Soleil) and ID20 (ESRF) beamlines. Measurements on CRISTAL were done using a Ge (2 20) crystal analyzer with $0.005^{\circ}$ mosaicity, and the wavelength was set to $2.09 \AA(E=5.9 \mathrm{keV})$. Measurements on ID20 were performed using a $\mathrm{LiF}$ (440) analyzer with a $0.05^{\circ}$ mosaicity, and a $1.007 \AA$ wavelength $(E=12.31 \mathrm{keV})$. Apart from the precise positions of the CDW peaks, we also measured the Bragg peaks during our experiment on ID20, recording 2D reciprocal space maps around 204 and 202 Bragg peaks.

Neutron diffraction experiments were performed on the "super-6T2" 4 circles diffractometer at LLB/Orphée reactor in Saclay, France, using a 2D detector [19] in order to measure full 3D reciprocal space maps. The temperature control was achieved through a displex cryocooler. The $\lambda / 2$ and $\lambda / 3$ harmonics of the normal wavelength $\lambda=2.345 \AA$ were filtered using PG crystals. Filtering was complete in the case of $\lambda / 2$, but a small signal coming from $\lambda / 3$ was still present. We used a 50 seconds/image counting time given the low magnetic intensity expected. To check the presence of very weak peaks, we performed a Laplacian of Gaussian filtering of the data, using the resolution function of the apparatus [20]. This procedure enhances the signal to noise ratio by more than one order of magnitude.

\section{RESULTS}

\section{A. Sample growth and structural characterization}

We have grown three sets of samples onto $\mathrm{MgO}\left(\begin{array}{lll}0 & 0 & 1\end{array}\right)$ substrates for this study. $\mathrm{Cr}$ grows on $\mathrm{MgO}$ with the same epitaxy relations as other $3 d$ bcc metals: The $\mathrm{Cr}$ lattice is rotated by $45^{\circ}$ around the film normal with respect to that of $\mathrm{MgO}$. C-type samples are $\mathrm{Cr} / \mathrm{MgO} / \mathrm{Cr}$ trilayers with a thin barrier. U-type samples are similar to $\mathrm{C}$-type but with a thicker barrier. Finally R-type samples are reference single Cr layers, similar to the bottom $\mathrm{Cr}$ layer of the $\mathrm{U}$ - and C-type samples. We have varied the thicknesses of the different $\mathrm{Cr}$ layers in order to disentangle thickness effects from other mechanisms. All $\mathrm{MgO}$ (001) substrates were annealed at $600{ }^{\circ} \mathrm{C}$ for 20 minutes prior to sample growth in order to remove surface contamination. A 10-nm-thick MgO buffer layer was deposited onto the substrate, to act as a diffusion trap [21] for carbon atoms still adsorbed after substrate annealing. The bottom $\mathrm{Cr}$ layers of $\mathrm{C}$ - and U-type samples, and the $\mathrm{Cr}$ layer of R-type samples were deposited at $100^{\circ} \mathrm{C}$ onto the $\mathrm{MgO}$ buffer and subsequently annealed at $500{ }^{\circ} \mathrm{C}$ for 30 minutes in order to improve crystalline quality and lower surface roughness.

The MgO barrier continuity and the defects in the barrier are crucial points that have to be carefully examined for this study since they can have a dramatic influence on the tunneling process. Several experimental results show that the $\mathrm{MgO}$ films are continuous with a limited number of defects. First, the growth mode of $\mathrm{MgO}$ on $\mathrm{Cr}$ is layer by layer as figured out by RHEED intensity oscillations systematically observed (see Fig. 1 for an example) during the $\mathrm{MgO}$ growth on $\mathrm{Cr}\left(\begin{array}{lll}0 & 0 & 1\end{array}\right)$. This is not surprising since the $\mathrm{MgO}$ surface energy is small (around $1 \mathrm{~J} / \mathrm{m}^{2}$ ) and half the value of the $\mathrm{Cr}$ surface energy. This large difference between surface energies pushes the $\mathrm{MgO}$ to cover the whole surface, $\mathrm{Mg}$ and $\mathrm{O}$ atoms being extremely mobile even at room temperature [22]. One important behavior that has also to be noticed is the lack of misfit dislocations in the $\mathrm{MgO}$ barrier for layers $5 \mathrm{ML}$ thick or less. Indeed, the critical thickness for $\mathrm{MgO}$ plastic relaxation on $\mathrm{Cr}\left(\begin{array}{lll}0 & 0 & 1\end{array}\right)$ is 


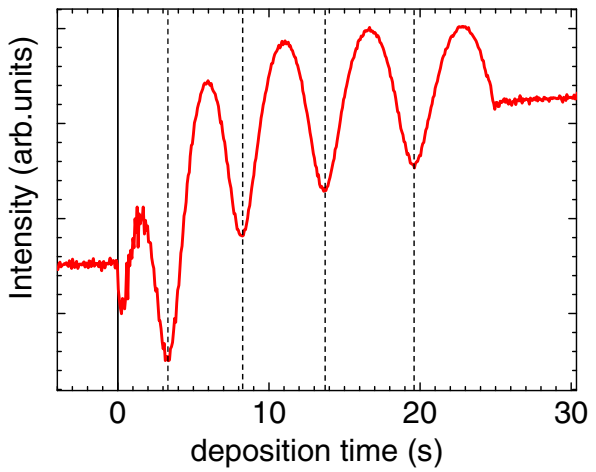

FIG. 1. (Color online) RHEED oscillations during $\mathrm{MgO}$ growth. Intensity of the $(00)$ peak recorded during the growth of the $\mathrm{MgO}$ barrier of sample $\mathrm{C} 2$. The vertical dashed line indicates the completion of $1,2,3$, or $4 \mathrm{ML}$ respectively, and the final $\mathrm{MgO}$ thickness is $4.75 \mathrm{ML}$. The presence of oscillations evidence a layer-by layer-growth, and are a strong indication of the absence of pinholes in the $\mathrm{MgO}$ barriers.

around $5 \mathrm{ML}$ as checked by measuring the in-plane lattice spacing variation during the growth using RHEED.

But other defects like pinholes (coming from dusts or defects in the substrate for example) may be present. To address this possibility, we performed a separate systematic study of the $\mathrm{MgO}$ barriers using conductive-tip atomic force microscopy (CT-AFM) images of $\mathrm{Cr} / \mathrm{MgO}$ (1.5-8 ML) bilayers. No electrical shunt (hot spot) characteristics of pinholes were observed for a large studied area (several $\mu \mathrm{m}^{2}$ ), as shown in Figs. 2(a)-2(c). This definitely proves that large size pinholes are not present in our $\mathrm{MgO}$ barrier. Figure 2(d) displays a typical example of $I(V)$ curve measured locally by CT-AFM on a $\mathrm{Cr}$ film covered by two monolayers of $\mathrm{MgO}$. The behavior is nonlinear as expected for tunnel transport. Moreover, we were able to create pinholes intentionally by increasing the force applied by the AFM tip on the $\mathrm{MgO}$ film: In this case, the $I(V)$ curve eventually becomes ohmic, and the resistance on the tip is four orders of magnitude smaller than that of the $\mathrm{MgO}$ film [and hence negligible on the curve displayed in Fig. 2(d)]. Finally, Fig. 2(e) gives a synthesis of our measurements by plotting the mean logarithmic resistance (measured at $1 \mathrm{~V}$ ) as a function of the $\mathrm{MgO}$ thickness. The resistance increases exponentially with $\mathrm{MgO}$ thickness, as expected for tunnel transport.

We can thus eliminate the presence of large pinholes, yet this CT-AFM study does not exclude the possibility to get small pinholes at atomic scale. This is unlikely though, considering the case of $\mathrm{Fe} / \mathrm{MgO} / \mathrm{Fe}$ trilayers (a very close system in terms of epitaxial growth). In this system, an antiferromagnetic coupling between $\mathrm{Fe}$ layers is observed $[18,35]$ for $\mathrm{MgO}$ thicknesses in the range 1.2 to 3 MLs. The magnetizations of the two Fe electrodes are found to be parallel for $\mathrm{MgO}$ thicknesses lower than a monolayer, due to the connection between the $\mathrm{Fe}$ electrodes via the uncompleted $\mathrm{MgO}$ atomic layer. However, for $\mathrm{MgO}$ thicknesses higher than $1 \mathrm{ML}$, a tunnel antiferromagnetic coupling is observed using SQUID or VSM measurements on $\mathrm{mm}^{2}$ size samples. This is clear proof that the number of pinholes, even of small sizes, is extremely reduced in this system, even at the millimeter scale. As $\mathrm{Cr}$ and
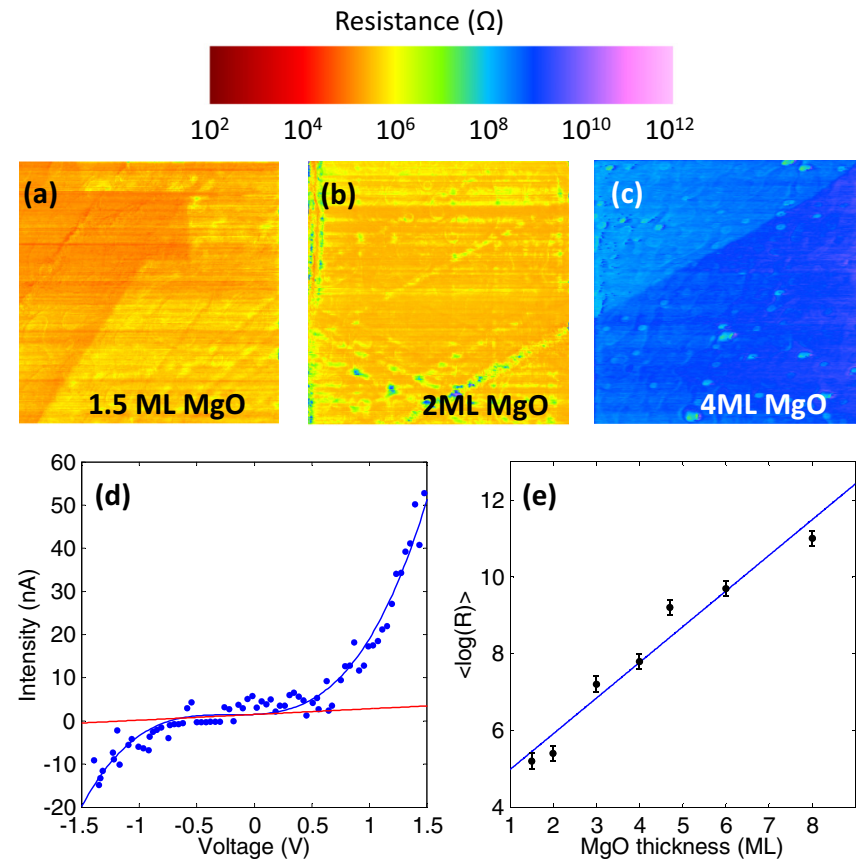

FIG. 2. (Color online) Conductive-tip AFM of $\mathrm{Cr} / \mathrm{MgO}$ bilayers. (a)-(c) Typical maps obtained on $3 \times 3 \mu \mathrm{m}^{2}$ areas for $\mathrm{MgO}$ thicknesses of $1.5,2$, or $4 \mathrm{ML}$ respectively. Note the absence of pinholes on the images. (d) Typical local $I(V)$ curve obtained on a $\mathrm{Cr} / \mathrm{MgO}$ (2 ML) bilayer by CT-AFM. The red line correspond to the linear fit of the low voltage region, and the blue line is a guide for the eyes. Note the marked nonlinearity for voltages exceeding $\pm 0.75 \mathrm{~V}$. (e) Evolution of the mean logarithmic resistance (measured at $V=1 \mathrm{~V}$ ) as a function of the $\mathrm{MgO}$ barrier thickness.

$\mathrm{Fe}\left(\begin{array}{ll}0 & 0\end{array}\right)$ surfaces are very similar (similar surface energies, similar lattice constant), there is no reason for having more defects in $\mathrm{MgO}$ between $\mathrm{Cr}$ layers than between Fe layers.

After the growth of the $\mathrm{MgO}$ barriers, the to-be-trilayers samples were cooled down so that the top $\mathrm{Cr}$ layers of $\mathrm{C}$ and U-type samples were deposited at $100^{\circ} \mathrm{C}$. We did not anneal the sample after deposition of the top $\mathrm{Cr}$ layers since our experience on $\mathrm{Fe} / \mathrm{MgO} / \mathrm{Fe}$ trilayers shows that heating the stack above $200{ }^{\circ} \mathrm{C}$ is detrimental to the tunnel coupling. All samples were capped with a $10 \mathrm{~nm}$ thick $\mathrm{MgO}$ layer to prevent oxidation. The thickness of each layer was checked after growth both by x-ray reflectivity and TEM (see Table I).

We have studied all samples using high-resolution transmission microscopy (HRTEM). Our images cover a significant portion of the samples, allowing for a detailed analysis representing the entirety of these samples. Low magnification images show the very low roughness of the different layers, and the continuity of the $\mathrm{MgO}$ barrier. Figure 3 presents HRTEM cross sections of two $\mathrm{Cr} / \mathrm{MgO} / \mathrm{Cr}$ trilayers studied along the [1 00 0] zone axis of $\mathrm{MgO}$. These micrographs confirm the high quality of the epitaxial growth of the trilayer in the [0 01 1] direction. The interface appears perfectly flat without any noticeable interface phase at the atomic scale.

In order to measure the local displacement and strain fields in our samples, we have applied the geometric phase method [23] on different HRTEM micrographs. Figures 3(b),(c) and 3(e),(f) display the strain fields in the 
TABLE I. Thickness of each layer of the eight samples used in the present study, as determined from x-ray reflectivity and TEM measurements. All $\mathrm{MgO}$ capping layers (omitted in the table) are 10 -nm thick. One monolayer of $\mathrm{MgO}$ is $0.2105 \mathrm{~nm}$ thick, so $C$-type samples correspond to 4 or $5 \mathrm{ML}$, and $U$-type samples correspond to 8 or $11 \mathrm{ML}$.

\begin{tabular}{lcccc}
\hline \hline Sample & & $\begin{array}{c}\text { bottom Cr } \\
\text { layer (nm) }\end{array}$ & $\begin{array}{c}\mathrm{MgO} \text { barrier } \\
(\mathrm{nm})\end{array}$ & $\begin{array}{c}\text { top Cr } \\
\text { layer (nm) }\end{array}$ \\
\hline Trilayers with thin & $\mathrm{C} 1$ & 200 & 0.8 & 100 \\
barriers & $\mathrm{C} 2$ & 131 & 1 & 78 \\
& $\mathrm{C} 3$ & 120 & 1 & 54 \\
\hline Trilayers with thick & $\mathrm{U} 1$ & 240 & 1.8 & 80 \\
barriers & $\mathrm{U} 2$ & 134 & 1.8 & 43 \\
& $\mathrm{U} 3$ & 122 & 2.3 & 38 \\
\hline Reference layers & $\mathrm{R} 1$ & 200 & & \\
& $\mathrm{R} 2$ & 85 & & \\
\hline \hline
\end{tabular}

$\mathrm{Cr} / \mathrm{MgO} / \mathrm{Cr}$ trilayers based on the HRTEM images of Figs. 3(a) and $3(\mathrm{~d})$. For the $\mathrm{C} 1$ sample, the $\varepsilon_{x x}$ color map [in-plane deformation calculated relative to Cr, see Fig. 3(b)] contains one single blue strip that covers the entire thickness of the trilayer, indicating a uniform strain field across the three layers in this direction. Moreover, none of the images recorded on this sample reveal any dislocation in the $\mathrm{MgO}$ barrier, while in the case of an unstrained $\mathrm{MgO}$ barrier on a $\mathrm{Cr}$ layer, the periodicity of dislocations should be $\sim 3 \mathrm{~nm}$. Hence no plastic relaxation occurs and the in-plane compression of $\mathrm{MgO}$ is given by the lattice mismatch, i.e., $\varepsilon_{x x}=-3.4 \%$. The situation is different for U-type samples: In this case the $\mathrm{MgO}$ thickness is larger than the critical value at which plastic relaxation occurs. Figure 3(d) shows dislocations in the $\mathrm{MgO}$ barrier, of which corresponding strain field are visible on Fig. 3(e-f). The $\varepsilon_{y y}$ maps (out-of-plane deformation) all reveal a sharp color change at the bilayer interface, as expected from the large difference of lattice parameters between $\mathrm{Cr}$ and $\mathrm{MgO}$ (remember that the $a_{\mathrm{MgO}} \simeq \sqrt{2} a_{\mathrm{Cr}}$ ). The out-of-plane deformation is difficult to measure precisely on these images, since the average is only done on a few $\mathrm{MgO}$ planes.

Since the magnetic structure of $\mathrm{Cr}$ is extremely sensitive to strain [13], we have also studied the structural properties of our samples with high resolution x-ray diffraction (XRD). Figures 4(a) and 4(b) display examples of raw data, obtained at low temperature. The $\mathrm{Cr} 004$ and $\mathrm{Cr} 202$ Bragg positions of the trilayers correspond to double peak structures, the more intense one (corresponding to the bottom layer which is thicker) matching almost exactly the Bragg peak of the $\mathrm{Cr}$ reference layer. We have determined the stress applied to the $\mathrm{Cr}$ layers on selected samples, using the sine square $\Psi$ method (see Supplemental Material for details [24]). The results, displayed in Fig. 4(c), evidence a sharp difference between bottom and top $\mathrm{Cr}$ layers: While the former experiences a small compressive stress (and hence a small tetragonal distortion, with $a<c$ ), not very different from the one observed in $\mathrm{Cr}$ reference samples, all top $\mathrm{Cr}$ layers exhibit high tensile stress (in this case $a>c$ ). The origin of this significant strain and stress for the top $\mathrm{Cr}$ layers is to be found in the presence of a large number of point defects, which is expected since these layers are not annealed. The small compressive strain of bottom $\mathrm{Cr}$ layers looks surprising at first, since lattice mismatch should favor a tensile strain.
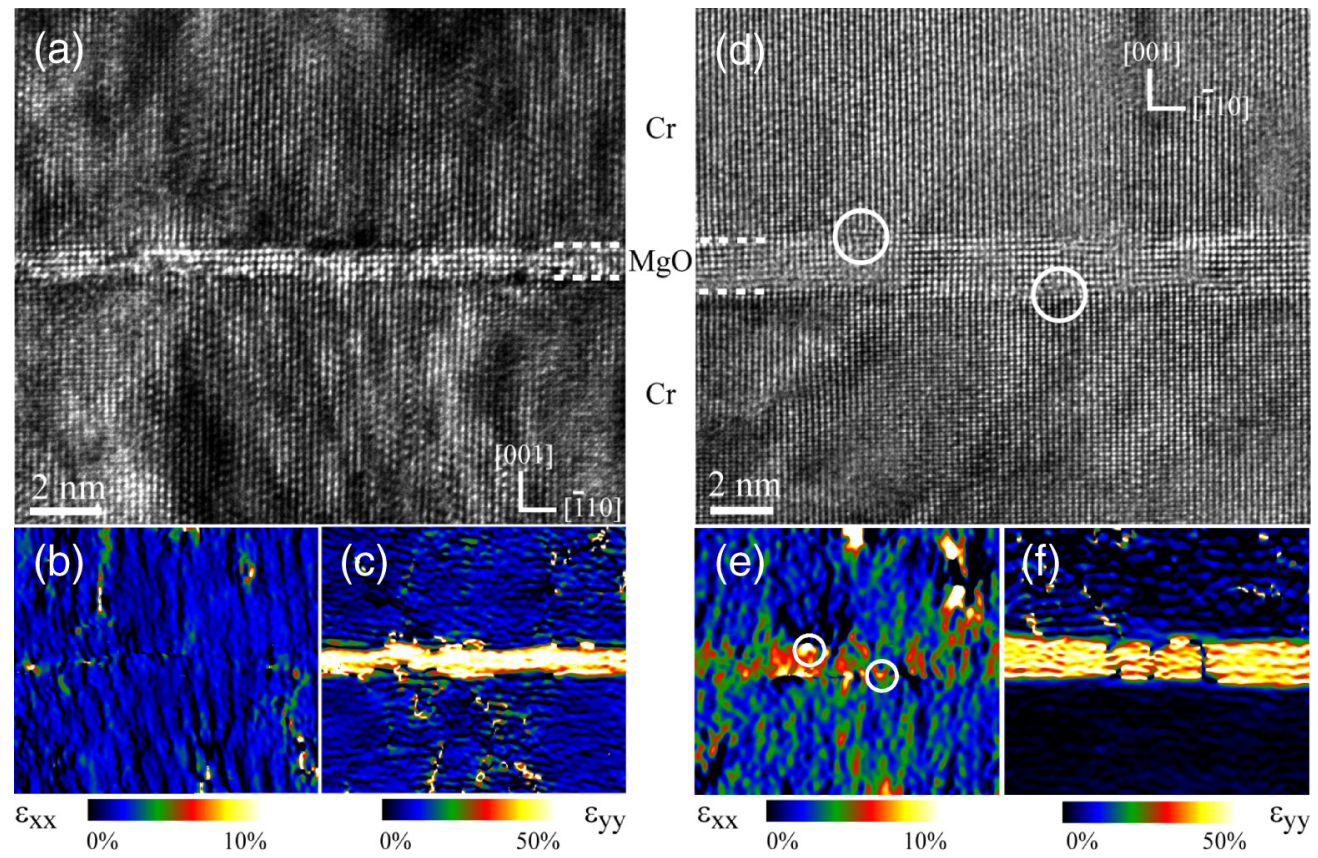

FIG. 3. (Color online) Cross sections of epitaxial $\mathrm{Cr} / \mathrm{MgO} / \mathrm{Cr}$ trilayers. (a) High resolution transmission electron micrograph of the $\mathrm{Cr} / \mathrm{MgO} / \mathrm{Cr}$ trilayer comprising a $4 \mathrm{ML}$ thick $\mathrm{MgO}$ barrier (sample $\mathrm{C} 1$ ). (b) In-plane strain $\left(\varepsilon_{x x}\right)$ calculated from phase images, relatively to the in-plane lattice parameter of the $\mathrm{Cr}$ layers. The contrast variations are not significant, so this image evidences the fully strained character of the $\mathrm{MgO}$ barrier. (c) Out-of-plane strain $\left(\varepsilon_{y y}\right)$ calculated from phase images. (d) $\mathrm{Cr} / \mathrm{MgO} / \mathrm{Cr}$ trilayer comprising a $8 \mathrm{ML}$ thick $\mathrm{MgO}$ barrier (sample U2). Note the presence of defects in the $\mathrm{MgO}$ barrier, due to its relaxation (the circles indicate dislocations). (e),(f) In- and out-of-plane deformation, as in (b) and (c). Note the high strain in (e), corresponding to the dislocations shown in (d). 


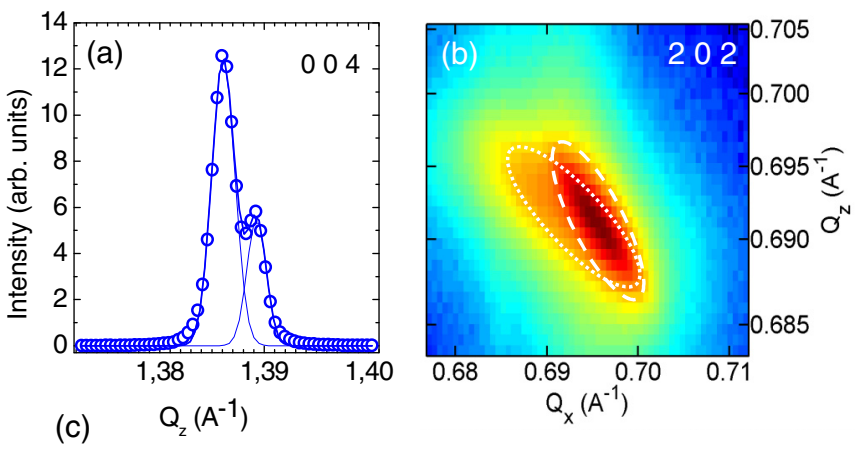

\begin{tabular}{|l|l|l|}
\hline \multirow{2}{*}{ Sample } & \multicolumn{3}{|l|}{ Equivalent stress at low temperature (GPa) } \\
\cline { 2 - 3 } & Bottom Cr layer & Top Cr layer \\
\hline C2 & -0.08 & 1.8 \\
\hline U3 & -0.2 & 0.7 \\
\hline R2 & -0.02 & - \\
\hline
\end{tabular}

FIG. 4. (Color online) Lattice parameters and equivalent stress in the $\mathrm{Cr} / \mathrm{MgO} / \mathrm{Cr}$ trilayers at low temperature. (a) Example of XRD $Q_{z}$ scans of a $\mathrm{Cr} / \mathrm{MgO} / \mathrm{Cr}$ trilayer (sample $\mathrm{C} 2$ at $72 \mathrm{~K}$ ) at the $\mathrm{Cr} 004$ Bragg peak of Cr. Solid lines correspond to the Gaussian fit of the data. (b) Example of reciprocal space map around a Bragg position different from the film normal ( 202 peaks of $\mathrm{Cr}$ sample U3 at $57 \mathrm{~K}$ here). The color scale corresponds to a logarithmic scale, and the white ellipses are guide for the eyes, showing the two peaks corresponding to the two different $\mathrm{Cr}$ layers. (c) Equivalent stress at low temperature, calculated using the sine square $\Psi$ method. The large positive stress in the top Cr layers corresponds to tensile strain $(a>c)$, while the bottom and reference layers exhibit a slight compressive strain $(a<c)$. The different behavior of bottom and top $\mathrm{Cr}$ layers comes from the lack of annealing of the top layers.

However, we can explain this apparent discrepancy by the difference of thermal expansion coefficients between $\mathrm{Cr}$ and $\mathrm{MgO}$ : The effect of annealing is to obtain nearly unstrained $\mathrm{Cr}$ layers at the annealing temperature, yet since the thermal expansion coefficient of $\mathrm{MgO}$ is larger than that of $\mathrm{Cr}$, the $\mathrm{MgO}$ substrate/Cr layer system contracts much more during cooling than would isolated $\mathrm{Cr}$. The strain state of all trilayers (both $U$ and $C$ type) taken as a whole is thus similar, but within each trilayer the bottom and top Cr layers are in very different strain states.

\section{B. Spin density waves in $\mathrm{Cr} / \mathrm{MgO} / \mathrm{Cr}$ trilayers}

We performed neutron diffraction experiments on the super-6T2 4 circles diffractometer at LLB/Orphée reactor in Saclay, France. We used a 2D detector [19] in order to record full 3D reciprocal space maps around the Bragg positions of interest. Neutron scattering is well suited for our study since it gives direct access to the magnetic structure by looking at and around the 100 and $001 \mathrm{Bragg}$ positions (forbidden for nuclear scattering): The commensurate phases give signals at $100 \mathrm{and} /$ or 001 , whereas the incommensurate SDW is evidenced by satellite peaks around 100 and 001 , depending

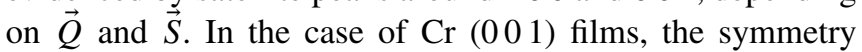
breaking gives rise to seven different magnetic phases, each having a unique neutron diffraction signature (see Table II).
TABLE II. (Color online) Magnetic Bragg peaks observed for the different magnetic phases of $\mathrm{Cr}$ thin films. The magnetic Bragg peaks are located at $\vec{Q}$ from the structural reflections, so they appear as pairs of satellites situated at $\delta$ from the structurally forbidden reflections. The SDW can be either commensurate (C) or incommensurate, and in the latter case longitudinal (L) or transverse (T). For thin films one also has to precise whether the spins lie in the film plane or not. All the phases except $\mathrm{L}_{\uparrow}$ and $\mathrm{C}_{\uparrow}$ consist of two equivalent $\vec{Q}$ or $\vec{S}$ domains. For each phase, the subscript designates the direction of the spins, while the superscript corresponds to the orientation of the propagation vector.

\begin{tabular}{|c|c|c|c|c|c|c|}
\hline Phase & $1 \pm \begin{array}{lll}\delta & 0 & 0\end{array}$ & $10 \pm \delta$ & 100 & $\pm \delta 0$ & $\begin{array}{lll}0 & 0 & 1 \pm \delta \\
\end{array}$ & $\begin{array}{lll}0 & 01 \\
\end{array}$ \\
\hline $\mathrm{T}_{\rightarrow}^{\text {out }}$ & & & & & & \\
\hline & & & & & & \\
\hline $\mathrm{T}_{\rightarrow}^{\text {in }}$ & 0 & & & 0 & & \\
\hline & & & & & & \\
\hline & & & & & & \\
\hline $\mathrm{C}_{\rightarrow}$ & & & 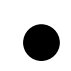 & & & \\
\hline $\mathrm{C}_{\uparrow}$ & & & 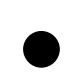 & & & \\
\hline
\end{tabular}

Typical neutron data (2D slices of the 3D reciprocal space map) are displayed in Figs. 5, 6, and 7. Data were recorded between 20 and $250 \mathrm{~K}$, but we present here only data obtained at $60 \mathrm{~K}$ for the sake of clarity.

The reference samples display a bulklike behavior: The map around 100 [Fig. 5(b)] shows only two peaks of similar intensities at $10 \pm \delta$, with $\delta \simeq 0.047$ similar to the bulk value. In Fig. 5(c), we see also two peaks, of inequivalent intensities, which is an artefact due to impurities within the substrate. We thus observe one single propagation vector, oriented along the film normal as already reported by other studies on $\mathrm{Cr}$ thin films [7], and as expected from the strain state measured by XRD [25]. The magnetic structure stays essentially the same in the whole temperature range we studied.

The behavior of the trilayers with thick barrier is more complex, as depicted in Fig. 6: In all U-type samples, we observe the coexistence of incommensurate and commensurate phases, the latter evidenced by the presence of peaks at 100 and 001 . The three peaks observed in Fig. 6(a) are not aligned: The $10 \pm \delta$ peaks position correspond to relaxed $\mathrm{Cr}$ (as for reference samples, $\delta$ is similar to the bulk value), while the commensurate peak location corresponds to $\mathrm{Cr}$ under large tensile stress (the in-plane deformation is about $1 \%$ ). We can thus attribute commensurate and incommensurate phases to the two different $\mathrm{Cr}$ layers, our observations being consistent with the strain states of each layer determined by XRD. Since no signal is observed at 001 , we can tell that the spins in the commensurate phase are perpendicular to the film plane. This coexistence of incommensurate and commensurate phases is 


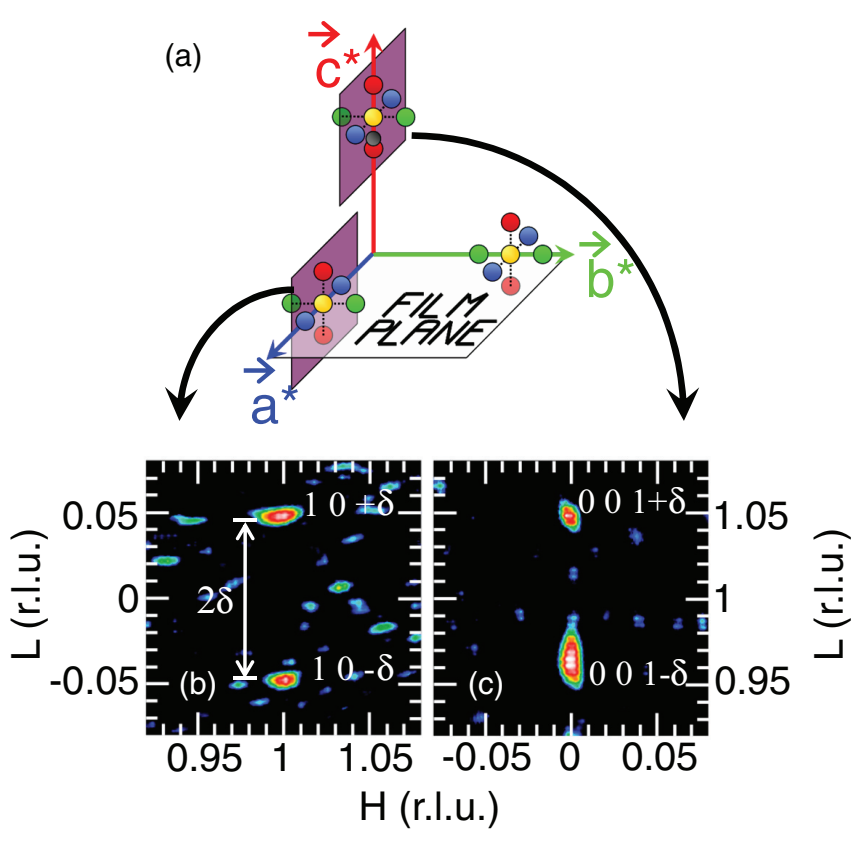

FIG. 5. (Color online) Reciprocal space maps obtained from neutron diffraction data, reference layer. (a) Schematic drawing of the $\mathrm{Cr}$ reciprocal lattice, showing the commensurate peaks (yellow), the incommensurate satellites (red, green, and blue) and a parasitic signal due to impurities in the substrate (black). The maps correspond to $(H 0 L)$ planes around 100 (left panels) and 001 (right panels). All maps were obtained from measurements at $60 \mathrm{~K}$. (b), (c) Reference layer (sample R1). The sample is single $\vec{Q}$, with a SDW period similar to bulk. We observed no commensurate peak. The intensity difference between the two peaks in (c) is an artifact due to impurities within the $\mathrm{MgO}$ substrate.

independent of temperature in the range studied and is expected given the strain state of the sample, as we shall see later.

The situation is strikingly different for trilayers comprising thin $\mathrm{MgO}$ barriers (C-type samples). Indeed, we observe four families of peaks at or around the 100 position [see Fig. 7(a)]: apart from the 100 and $10 \pm \delta$ peaks already observed for the three U-type samples, we see a pair of weak additional

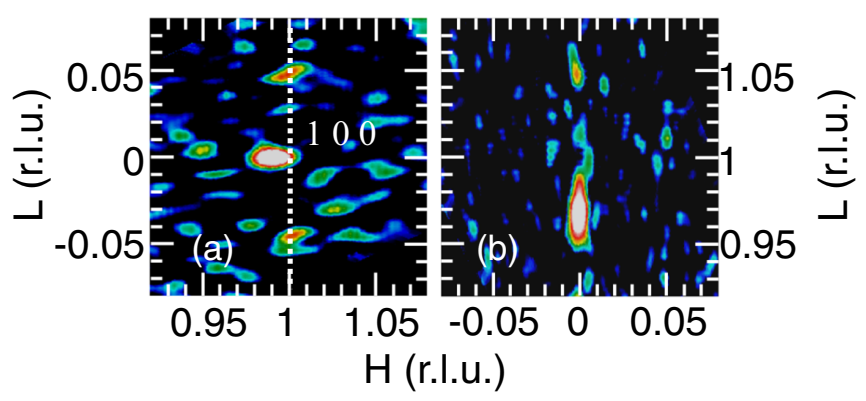

FIG. 6. (Color online) Reciprocal space maps obtained from neutron diffraction data, trilayer with thick $\mathrm{MgO}$ barrier (sample $\mathrm{U} 3)$, at $60 \mathrm{~K}$. (a) $(\mathrm{HOL})$ plane around 100 . (b) $(\mathrm{HOL})$ plane around 001 . Along with the bulklike incommensurate peaks already seen for the reference, we observe a rather strong commensurate peak which corresponds to a different in-plane lattice parameter (see dotted line in (a).

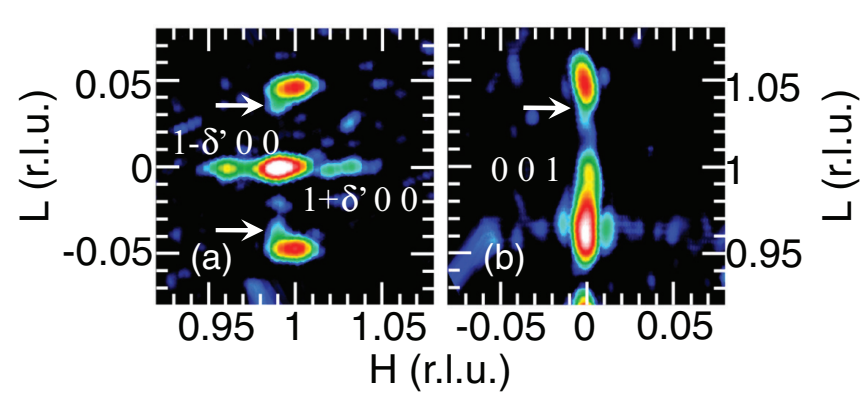

FIG. 7. (Color online) Reciprocal space maps obtained from neutron diffraction data, trilayer with thin $\mathrm{MgO}$ barrier (sample $\mathrm{C} 2$ ), at $60 \mathrm{~K}$. (a) $(H 0 L)$ plane around 100 . (b) $(H 0 L)$ plane around 001. We evidence two anomalous SDW phases. The first has a propagation vector along $\vec{a}^{\star}$, as evidenced by the $1 \pm \delta^{\prime} 00$ peaks seen in (a). The second anomalous phase is evidenced by the small shoulders (indicated by the white arrows) in (f), corresponding to $10 \pm \delta^{\prime \prime}$. The $001+\delta^{\prime \prime}$ peak is also present, even if it is more difficult to separate from $001+\delta$ given the lower resolution of the diffractometer in the $\vec{c}^{\star}$ direction in this configuration.

satellites at $1 \pm \delta^{\prime} 00$, with $\delta^{\prime} \simeq 0.035$ (a value significantly smaller than $\delta$ ). The barycenter of these two peaks does not correspond to the commensurate peak (indeed the $1-\delta^{\prime} 00$ peak is not fully separated from the commensurate peak on Fig. 7(b), contrary to the $1+\delta^{\prime} 00$ peak). The map around 001 [Fig. 7(b)] shows no peak at $\pm \delta^{\prime} 01$. This indicates that the spins of $\mathrm{Cr}$ atoms in this anomalous SDW phase are oriented along the film normal, and it thus corresponds to a $T_{\uparrow}^{\text {in }}$ phase according to the notations of Table II. However, we observe a peak at 001 , so a commensurate phase with spins in the film plane is present, contrary to the U-type samples. The peak at 001 is much weaker than the peak at 100 , so the main commensurate phase is $C_{\uparrow}$, with spins pointing out of the film plane.

We also observe other very weak peaks (indicated by the white arrows in Fig. 7), corresponding to a second anomalous propagation vector, along $\vec{c}^{\star}$ : Given the resolution in these two configurations, these $10 \pm \delta^{\prime \prime}$ and $001+\delta^{\prime \prime}$ peaks appear as shoulders of the more intense $10 \pm \delta$ and $001+\delta$ ones corresponding to the bulklike phase. Therefore these peaks correspond to a transverse SDW phase with in-plane spins (or $T_{\rightarrow}^{\text {out }}$ ); however, it is difficult to give a value of $\delta^{\prime \prime}$ based on the sole neutron diffraction measurements. Both anomalous phases disappear at high temperature, the broad transition taking place between 120 and $180 \mathrm{~K}$. In this temperature range, we also observe a significant increase of the intensities of the magnetic Bragg peaks, which is consistent with an incommensurate/commensurate transition.

\section{Charge density wave in $\mathrm{Cr} / \mathrm{MgO} / \mathrm{Cr}$ trilayers}

In order to measure precisely the period of the different SDW phases evidenced by neutrons, we have also studied the charge density wave (CDW) peaks associated to the SDW in the three types of samples. To do so, we performed high resolution XRD measurements using the six circles diffractometers of the CRISTAL and ID20 beamlines at the SOLEIL and ESRF synchrotron sources. The CDW gives rise to very weak satellite peaks around the allowed Bragg peaks of the bcc structure (the incommensurate CDW peaks are located 

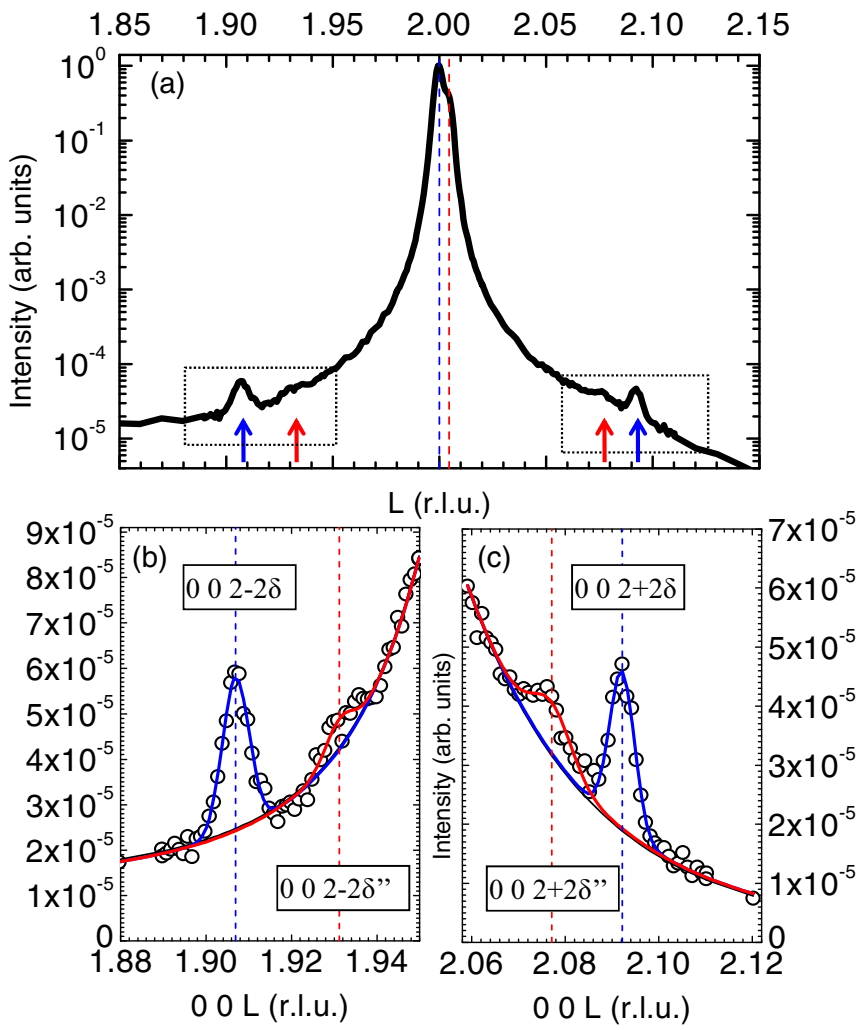

FIG. 8. (Color online) Observation of CDW peaks in $\mathrm{Cr} / \mathrm{MgO} / \mathrm{Cr}$ trilayers. (a) Synchrotron XRD scan along the $00 \mathrm{~L}$ direction of the C2 trilayer, obtained on the ID20 beamline at the ESRF. The general scan shows the split Bragg peak at 002 and the two pairs of CDW satellites, indicated by the red and blue arrows. The position of the more intense component of the Bragg peak, corresponding to the bottom $\mathrm{Cr}$ layer, is used to normalize the distance and is thus set as $L=2$. The data were recorded at $72 \mathrm{~K}$. (b), (c) Zoom on the low and high $L$ parts of the scan, represented by the two boxes on (a). The red and blue solid lines correspond to the Gaussian fits used to determine the CDW peaks positions. The vertical dotted lines correspond to the value obtained from the fits. One clearly sees that the barycenters of the two series of peaks are different, which means that the two CDW phases do not correspond to the same $\mathrm{Cr}$ layer.

at the end of the tail of the Bragg peaks, whose intensity is about $10^{4}$ to $10^{5}$ times larger). Hence this technique can give very precise values of the peak positions (accuracy is better than $10^{-4}$ reciprocal lattice units in our case), but it is not sensitive to commensurate phases.

We observe the CDW peaks corresponding to the bulklike SDW for all types of samples, with periods very slightly larger than for bulk (at low temperature, $\delta$ is 0.0447 for sample R2, 0.0466 for sample U3 and 0.0463 for sample C2, compared to 0.04838 for bulk $\mathrm{Cr}$ [13]). While we observe no other peaks for the U- and R-type samples (see Supplemental Material for details [24]), the situation is again different for the C-type sample we looked at, as shown in Fig. 8: The $002 \pm 2 \delta^{\prime \prime}$ peaks are now clearly visible. The very high accuracy of the XRD measurements allow us both to give a precise value of $\delta^{\prime \prime}=$ 0.0365 , and to determine to which layer each of the two SDW corresponds. The center of gravity of the two $002 \pm 2 \delta$ peaks corresponds to $L=2$, i.e., the bottom annealed layer we use (a)
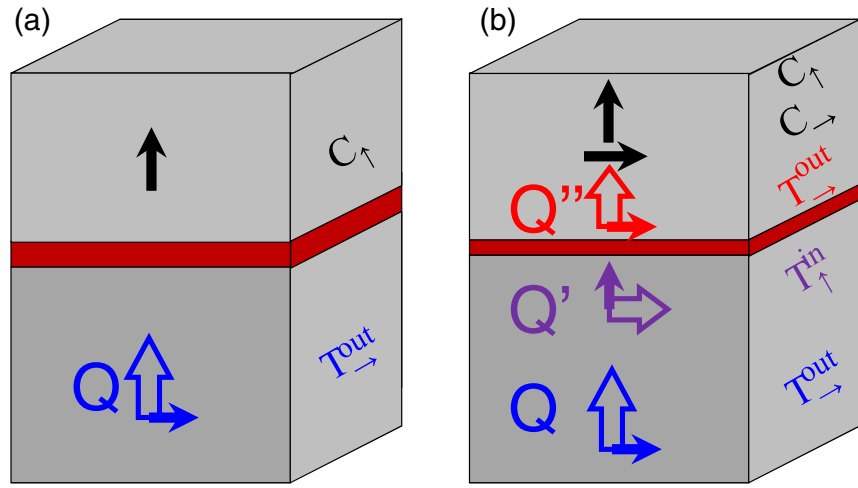

FIG. 9. (Color online) Magnetic ordering in $\mathrm{Cr} / \mathrm{MgO} / \mathrm{Cr}$ trilayers. (a) Magnetic structure of the U-type samples. The top $\mathrm{Cr}$ layer, highly strained, exhibits a commensurate structure, with out-of-plane spins. (b) Magnetic structure of the C-type samples. The spins in the commensurate phase located in the top $\mathrm{Cr}$ layer are now in-plane, and two anomalous SDW phases are observed. The different lengthes of the propagation vectors correspond to the values observed for $\delta, \delta^{\prime}$, and $\delta^{\prime \prime}$.

to scale the length in the reciprocal space. On the contrary, the center of the two $002 \pm 2 \delta^{\prime \prime}$ peaks corresponds to $L=2.004$, i.e., the top $\mathrm{Cr}$ layer. For practical reasons, we were not able to observe the XRD counterparts of the $1 \pm \delta^{\prime} 00$ observed in neutron diffraction: The 002 Bragg peak is significantly broader in the $\vec{a}^{\star}$ direction compared to the $\vec{c}^{\star}$ direction, so the background is increased and no CDW peak could be detected.

\section{DISCUSSION}

\section{A. Strain and magnetic properties of $\mathrm{Cr} / \mathrm{MgO} / \mathrm{Cr}$ trilayers}

By combining the neutron and $\mathrm{x}$-ray diffraction data, we are able to establish a schematic picture of the magnetic ordering of our $\mathrm{Cr} / \mathrm{MgO} / \mathrm{Cr}$ trilayers, displayed in Fig. 9. Since there is no significant broadening of the $1 \pm \delta^{\prime} 00$ peaks seen with neutrons and of the $002 \pm 2 \delta^{\prime \prime}$ seen with $\mathrm{x}$ rays, we can tell that the size of the domains corresponding to these phase is larger than $10 \mathrm{~nm}$ (the typical beam coherence length). Stress and the presence of defects are well known to have a dramatic influence on the magnetic ordering of chromium. Indeed, we can fully explain the magnetic behavior of $\mathrm{U}$ - and R-type samples in this framework. The single- $\vec{Q}$ character of the main incommensurate phases is consistent with the strain state [25], $\vec{Q}$ being collinear with the elongated direction of the cubic unit cell determined by XRD. Defects also explain [26] the presence of a commensurate phase at low temperature in the top layer of the U-type samples: We know that the commensurate phases correspond to layers experiencing a large in-plane stress [see, e.g., Fig. 6(a)] and a high defect concentration, resulting from the lack of annealing.

However, we emphasize that the two anomalous SDW phases (associated to $\delta^{\prime}$ and $\delta^{\prime \prime}$ ) evidenced in trilayers with thin $\mathrm{MgO}$ barriers [C-type samples, Fig. 9(b)] can not be expected from the works already reported on isolated chromium thin films and are also not observed in U-type samples with thick $\mathrm{MgO}$ barriers. Such modifications of the SDW period (and 
hence of the $\delta$ value) are known to result from an alteration of the electronic structure, induced by strain $[13,25,27,28]$ and/or doping $[13,29]$. In the present case this alteration is large, since the value of $\delta^{\prime}$ and $\delta^{\prime \prime}$ we observe would correspond to a significant equivalent doping (e.g., $1 \%$ of $\mathrm{Mn}$ ) or a very large applied stress (2.3 GPa).

In our case, it is not possible to explain the value of $\delta^{\prime}$ by the stress we measured in the bottom Cr layers [see Fig. 4(c)]: Using $\mathrm{d} \ln Q / \mathrm{d} p=5 \cdot 5 \cdot 10^{-12} \mathrm{~Pa}^{-1}$, observed both for thin films and bulk samples [7], the observed value of $\delta^{\prime}$ should correspond to a stress of $2.3 \mathrm{GPa}$, more than one order of magnitude larger than the value we actually measured. Another possible explanation for the value of $\delta^{\prime}$, indirectly linked to strain, could be the presence of a lattice of misfit dislocations. Indeed, the lattice mismatch between $\mathrm{Cr}$ and $\mathrm{MgO}$ is $\simeq-3.4 \%$, so the period of the misfit dislocation lattice would be consistent with the value observed for $\delta^{\prime}$. We can also rule out this explanation since we did not observe satellite peaks corresponding to a lattice of misfit dislocation in any trilayer ( $\mathrm{C}$ or $\mathrm{U}$ type) in our synchrotron radiation diffraction experiments. Moreover, this rationale would lead to predicting the presence of a phase with a modified propagation vector for all samples, which we do not observe experimentally. We can thus rule out strain as the origin of the anomalous $\mathrm{T}_{\uparrow}^{\mathrm{in}}$ phase.

Strain can also be excluded as the origin of the other anomalous phase: The value of $\delta^{\prime \prime}$ is consistent with the magnitude of the equivalent stress in the top $\mathrm{Cr}$ layers of C-type samples (2.3 GPa expected using the value of $\mathrm{d} \ln Q / \mathrm{d} p$ already mentioned, to compare with $1.8 \mathrm{GPa}$ measured on sample C2), but the sign is not: Indeed the large tensile stress we measured in these layers should favor an in-plane propagation vector, and not an out-of-plane one as we observe.

Direct thickness effects can also be ruled out in our case: Finite-size effects are very unlikely, since SDW quantization effects, if any, should be small in the present case given the large number of $\mathrm{Cr}$ planes in our films (at least 300). More generally, the thickness of the Cr layers seems not to be a relevant parameter in our case: The presence of incommensurate phases with anomalous propagation vectors appears uncorrelated with the $\mathrm{Cr}$ thicknesses, and depends solely on the thickness of the $\mathrm{MgO}$ barrier (for example the neutron diffraction reciprocal space maps of sample U1 look like Fig. 6, and not like Fig. 7 or the maps obtained on sample $\mathrm{C} 1$, not shown here).

\section{B. Electronic structure of $\mathrm{Cr} / \mathrm{MgO} / \mathrm{Cr}$ trilayers}

Our study demonstrates the crucial role of the $\mathrm{MgO}$ barrier (and of its thickness) for the magnetic behavior observed in $\mathrm{Cr} / \mathrm{MgO} / \mathrm{Cr}$ trilayers. As purely structural effects can be ruled out, this suggests that equilibrium tunnel transport through the $\mathrm{MgO}$ barrier may explain the specificities of thin barrier trilayers. Using the close link between the electronic structure and the magnetic ordering in $\mathrm{Cr}$, we can now propose a qualitative interpretation of the magnetic ordering of our system (in particular the presence of anomalous magnetic phases) in terms of tunnel coupling.

The stabilization of the spin density wave originates in the nesting properties of the Fermi surface [see Fig. 10(a)]: Large portions of the hole octahedron can be superimposed with
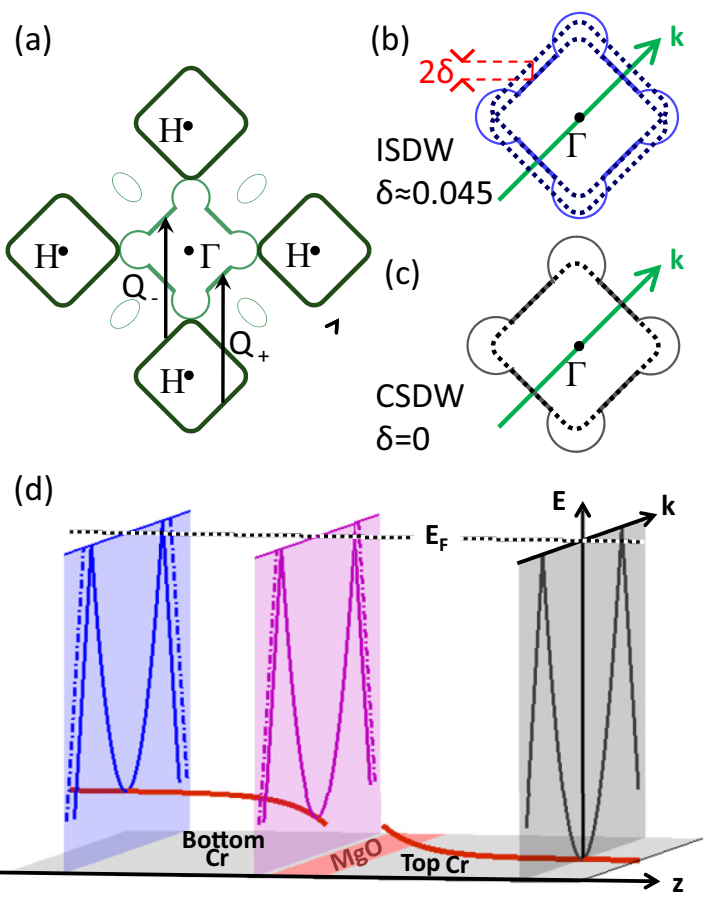

FIG. 10. (Color online) Schematic band structure in coupled $\mathrm{Cr} / \mathrm{MgO} / \mathrm{Cr}$ trilayers. (a) Schematic 2D [ $\left[\begin{array}{lll}0 & 0 & 1\end{array}\right]$ slice of the Fermi surface of bulk $\mathrm{Cr}$. The electron jack (comprising the electron octahedron) is centered at the $\Gamma$ point (light green). The hole octahedrons, centered at the $H$ point, are represented in dark green. $\vec{Q}_{+}$and $\vec{Q}_{-}$are examples of nesting vectors. (b) Nesting of the Fermi surface, incommensurate case. The hole octahedron is slightly larger than the electron octahedron; the $\delta$ parameter is nonzero. (b) Nesting of the Fermi surface, commensurate case $(\delta=0)$. (d) Schematic evolution of the band structure along the coupled $\mathrm{Cr} / \mathrm{MgO} / \mathrm{Cr}$ trilayers ( $z$ is along the trilayer normal). Far from the barrier, the dispersion along $\{110\}$ [indicated by the green arrows of (b) and (c)] exhibits the band folding characteristic of $\mathrm{Cr}$ antiferromagnetic ordering (commensurate in black, and incommensurate in blue and purple). Given the significant overlap of the $\Delta_{5}$ wave functions across the tunnel barrier, an "intermediate" band structure is present close to the $\mathrm{MgO}$ barrier. The bottom of the $\Delta_{5}$ bulk band, which exhibits band bending, is represented by the solid red line.

the electron octahedron (centered on the $\Gamma$ point of the first Brillouin zone) by a translation of vectors $Q_{ \pm}$incommensurate with the reciprocal lattice vectors [incommensurate SDW, Fig. 10(b)], or by a translation of a reciprocal lattice vector [commensurate SDW, Fig. 10(c)]. In nearly perfect $\mathrm{Cr}$ (as our bottom $\mathrm{Cr}$ layers), incommensurate nesting is favored, but a perturbation of the electronic structure by disorder (presence of defects), stress, or doping can favor commensurate nesting instead. In the case of $\mathrm{Cr}$ alloys, adding electron donors in the $\mathrm{Cr}$ matrix increases the SDW period and ultimately leads to a transition to a commensurate phase. This can be linked, in a rigid bands description, to an expansion of the electron pocket and a shrink of the hole pocket (equivalent to an increase of the Fermi level) which modifies the stabilized nesting vector.

In our thick barrier trilayers, the nesting vector is different (commensurate or incommensurate) in the top or bottom layers, because of their structural differences. In thin barrier trilayers, the anomalous SDW phases of incommensurability 
parameters $\delta^{\prime}$ and $\delta^{\prime \prime}$ correspond to a strong modification of the Fermi surface with respect to perfect bulk $\mathrm{Cr}$ and each of the two $\mathrm{Cr}$ layers, equivalent to an increase of the electronic concentration in pure $\mathrm{Cr}$ by a doping with $0.5 \% \mathrm{Mn}$.

In these $\mathrm{Cr} / \mathrm{MgO} / \mathrm{Cr}$ samples, the two $\mathrm{Cr}$ layers are not electronically independent when the $\mathrm{MgO}$ barrier thickness is five monolayers or less. Bulk Cr wave functions with $\Delta_{5}$ symmetry (linked to the SDW stabilization in $\mathrm{Cr}$ ) originating from one $\mathrm{Cr}$ layer persist as evanescent states in the $\mathrm{MgO}$ barrier [30] (with a rather low attenuation coefficient [31,32]) and may be transmitted partially to the other $\mathrm{Cr}$ layer where bulk wave functions with the same symmetry exist. At equilibrium, the tunnel transport of electrons in both directions leads to a significant overlap of the $\Delta_{5}$ bulk band located on both sides of the $\mathrm{MgO}$ barrier. Besides, resonant interface states of $\Delta_{1}$ and $\Delta_{5}$ symmetry are present at the $\mathrm{Cr} / \mathrm{MgO}$ interface [30] and shall amplify the electrons wave functions overlap through the $\mathrm{MgO}$ barrier. According to theoretical studies, these states may be transmitted without attenuation under resonance conditions $[31,33]$.

Coupling in FM/insulator/FM heterostructures in the model of Slonczewski [34] is based on the overlap of polarized wave functions through the tunnel barrier. This was applied to interpret the exchange coupling observed in $\mathrm{Fe} / \mathrm{MgO} / \mathrm{Fe}$ trilayers, where the interface resonant $\Delta_{1}$ state could be the main actor [35], through a resonant tunnel transport mechanism. In our $\mathrm{Cr} / \mathrm{MgO} / \mathrm{Cr}$ trilayers, the electronic perturbation at the interfaces arising from the connection of metallic wave functions from the bottom and the top layer through the barrier may induce the stabilization of magnetic phases with a larger modulation period (incommensurability parameters $\delta^{\prime}$ and $\delta^{\prime \prime}$ ) that we evidenced in thin MgO trilayers. These would be the signature of a magnetic coupling in our systems, and would be localized near the $\mathrm{Cr} / \mathrm{MgO}$ interfaces. For $\mathrm{Cr}\left(\begin{array}{lll}1 & 1 & 0\end{array}\right)$ and $\mathrm{Cr}\left(\begin{array}{lll}0 & 0 & 1\end{array}\right)$ surfaces, the magnetic ordering of $\mathrm{Cr}$ (anisotropy and phase) can indeed be modified on a significant thickness through interface effects $[7,9,36,37]$. In our case, the modification of the local magnetic order is more subtle as the incommensurability parameter has an intermediate value $\delta^{\prime} \approx \delta^{\prime \prime} \approx 0.035$ between that of the top $\delta=0$ and the bottom $\delta \approx 0.047$ layer. This is analogous to a doping gradient of a $\mathrm{Cr}$ alloy between the bottom, top layers, and the interfaces, which corresponds to an electronic concentration gradient in the system, as pictured in Fig. 10(d). Since the two Cr layers are not isolated, the Fermi level is unique for the whole system, and the effect of the $\Delta_{5}$ wave functions overlap is to generate band bending: Far from the interfaces, the width of the $\Delta_{5}$ bulk bands are different in the two phases [red line in Fig. 10(d)]. Thus there exists a zone of "intermediate band width," close to both $\mathrm{Cr} / \mathrm{MgO}$ interfaces.
In our case, given the magnetic energy cost of having a gradient of $\delta$ values, $\mathrm{Cr}$ tends to exhibit a unique, but altered, $\delta$ value near interfaces. We emphasize that the coupling we evidence through a modification of the magnetic anisotropy and of the magnetic period in the $\mathrm{Cr}$ layers has an electronic origin (and hence does not explain the orientations of the spins in the different magnetic phases, which goes beyond the scope of this paper).

We also point out that the critical $\mathrm{MgO}$ thickness above which there is no coupling is very similar to the one reported for the antiferromagnetic coupling observed when the metallic layers are ferromagnetic [38]. Besides, this critical thickness also corresponds to the beginning of plastic relaxation of $\mathrm{MgO}$, during which misfit dislocations appear in order to release the epitaxial strain [see Figs. 3(d) and 3(e)]. It has recently been shown that these dislocations have a dramatic impact on tunnel transport in magnetic tunnel junctions [39].

In summary, we have evidenced an unexpected behavior in well characterized $\mathrm{Cr} / \mathrm{MgO} / \mathrm{Cr}\left(\begin{array}{lll}0 & 0 & 1\end{array}\right)$ trilayers: When the $\mathrm{MgO}$ barrier is ultra thin, the magnetic states of the two AF Cr layers are no longer independent. This behavior can be explained neither by results already reported on $\mathrm{Cr}$ thin films nor by known strain effects as demonstrated through measurements of a reference $\mathrm{Cr}$ layer and trilayers having a thicker $\mathrm{MgO}$ barrier. However our experimental data can be understood thanks to a tunnel coupling between the two antiferromagnetic layers which can especially explain the observation of phases with altered propagation vectors. Such coupling can be amplified through interface resonant states exhibiting the same $\Delta_{5}$ symmetry. This tunnel coupling mechanism appears as a more general feature of epitaxial magnetic tunnel junctions even through an $\mathrm{MgO}$ barrier. Moreover, our work highlights that even though antiferromagnets have no net magnetization, their properties can be changed by coupling when included into multilayers. This opens routes for manipulating their magnetic ordering.

\section{ACKNOWLEDGMENTS}

We thank T. Robillard and J.-L. Meuriot for technical support at LLB, F. Greullet for her assistance during sample growth, C. Dufour, S. Robert, and N. Gonzales for their help during the XRD measurements at IJL, J. Chevreul and N. Guiblin for their help during the XRD measurements at SPMS, and J. Crestou and C. Crestou for TEM thin foil preparation. This work was supported by the French Agence Nationale de la Recherche, ANR-11-JS10-005 "Electra." The HRTEM experiments were supported by the METSA network.
[1] A. S. Nunez, R. A. Duine, P. Haney, and A. H. MacDonald, Phys. Rev. B 73, 214426 (2006).

[2] Y. Xu, S. Wang, and K. Xia, Phys. Rev. Lett. 100, 226602 (2008).

[3] S. Urazhdin and N. Anthony, Phys. Rev. Lett. 99, 046602 (2007).

[4] A. V. Kimel, B. A. Ivanov, R. V. Pizarev, P. A. Usachev, A. Kiriliuk, and Th. Rasing, Nat. Phys. 5, 727 (2009).
[5] M. N. Baibich, J. M. Broto, A. Fert, F. Nguyen Van Dau, F. Petroff, P. Etienne, G. Creuzet, A. Friederich, and J. Chazelas, Phys. Rev. Lett. 61, 2472 (1988).

[6] H. Zabel, J. Phys.: Condens. Matter 11, 9303 (1999).

[7] P. Sonntag, P. Bodeker, A. Schreyer, H. Zabel, K. Hamacher, and H. Kaiser, J. Magn. Magn. Mater. 183, 5 (1998). 
[8] R. S. Fishman, J. Phys.: Condens. Matter 13, R235 (2001).

[9] E. Kravtsov, R. Brucas, B. Hjörvarsson, A. Hoser, A. Liebig, G. J. McIntyre, M. A. Miliyaev, A. Nefedov, L. Paolasini, F. Radu, A. Remhof, V. V. Ustinov, F. Yakhou, and H. Zabel, Phys. Rev. B 76, 024421 (2007).

[10] E. E. Fullerton, J. L. Robertson, A. R. E. Prinsloo, H. L. Alberts, and S. D. Bader, Phys. Rev. Lett. 91, 237201 (2003).

[11] K. Mibu and T. Shinjo, J. Phys. D: Appl. Phys. 35, 2359 (2002).

[12] M. Takeda, K. Mibu, T. Shinjo, Y. Endoh, and J. Suzuki, Phys. Rev. B 70, 104408 (2004).

[13] E. Fawcett, Rev. Mod. Phys. 60, 209 (1988).

[14] A. Yeh, Y.-A. Soh, J. Brooke, G. Aeppli, T. F. Rosenbaum, and S. M. Hayden, Nature (London) 419, 459 (2002).

[15] R. Jaramillo, Y. Feng, J. C. Lang, Z. Islam, G. Srajer, P. B. Littlewood, D. B. McWhan, and T. F. Rosenbaum, Nature (London) 459, 405 (2009).

[16] R. K. Kummamuru and Y.-A. Soh, Nature (London) 452, 859 (2008).

[17] O. G. Shpyrko, E. D. Isaacs, J. M. Logan, Y. Feng, G. Aeppli, R. Jaramillo, H. C. Kim, T. F. Rosenbaum, P. Zschack, M. Sprung, S. Narayanan, and A. R. Sandy, Nature (London) 447, 68 (2007).

[18] C. Tiusan, F. Greullet, M. Hehn, F. Montaigne, S. Andrieu, and A. Schuhl, J. Phys.: Condens. Matter 19, 165201 (2007).

[19] A. Gukasov, A. Goujon, J.-L. Meuriot, C. Person, G. Exil, and G. Koskas, Physica B 397, 131 (2007).

[20] A. M. Bataille, V. Auvray, Ch. Gatel, and A. Gukasov, J. App. Cryst. 46, 726 (2013).

[21] M. Sicot, S. Andrieu, C. Tiusan, F. Montaigne, and F. Bertran, J. Appl. Phys. 99, 08D301 (2006).

[22] J. L. Vassent, A. Marty, B. Gilles, and C. Chatillon, J. Cryst. Growth 219, 444 (2000).

[23] E. Snoeck, M. J. Hytch, and R. Kilaas, Ultramicroscopy 74, 131 (1998). GPA software, www.hremresearch.com
[24] See Supplemental Material at http://link.aps.org/supplemental/ 10.1103/PhysRevB.90.035432 for the method used to determine the epitaxial strain, the equivalent stress, the way of calculating error bars, and also the display of the synchrotron XRD curves of two samples.

[25] Z. Barak and M. B. Walker, J. Phys. F: Metall. Phys. 12, 483 (1982).

[26] Z. Boekelheide, E. Helgren, and F. Hellman, Phys. Rev. B 76, 224429 (2007).

[27] G. E. Bacon and N. Cowlam, J. Phys. C: Solid State Phys. 2, 238 (1969).

[28] T. M. Rice, Phys. Rev. B 2, 3619 (1970).

[29] E. Fawcett, H. L. Alberts, V. Yu. Galkin, D. R. Noakes, and J. V. Yakhmi, Rev. Mod. Phys. 66, 25 (1994).

[30] M. A. Leroy, A. M. Bataille, F. Bertran, P. LeFevre, A. TalebIbrahimi, and S. Andrieu, Phys. Rev. B 88, 205134 (2013).

[31] P. H. Dederichs, Ph. Mavropoulos, O. Wunnicke, N. Papanikolaou, V. Bellini, R. Zeller, V. Drchal, and J. Kudrnovský, J. Magn. Magn. Mater. 240, 108 (2002).

[32] W. H. Butler, X.-G. Zhang, T. C. Schulthess, and J. M. MacLaren, Phys. Rev. B 63, 054416 (2001).

[33] O. Wunnicke, N. Papanikolaou, R. Zeller, P. H. Dederichs, V. Drchal, and J. Kudrnovsky, Phys. Rev. B 65, 064425 (2002).

[34] J. C. Slonczewski, Phys. Rev. B 39, 6995 (1989).

[35] C. Tiusan, M. Sicot, J. Faure-Vincent, M. Hehn, C. Bellouard, F. Montaigne, S. Andrieu, and A. Schuhl, J. Phys.: Condens. Matter 18, 941 (2006).

[36] E. Kravtsov, A. Nefedov, G. Nowak, K. Zhernenkov, H. Zabel, B. Hjorvarsson, A. Liebig, A. Hoser, G. J. McIntyre, L. Paolasini, and A. Remhof, J. Phys.: Condens Matter 21, 336004 (2009).

[37] E. Rotenberg, O. Krupin, and S. D. Kevan, New J. Phys. 10, 023003 (2008).

[38] J. Faure-Vincent, C. Tiusan, C. Bellouard, E. Popova, M. Hehn, F. Montaigne, and A. Schuhl, Phys. Rev. Lett. 89, 107206 (2002).

[39] F. Bonell, S. Andrieu, C. Tiusan, F. Montaigne, E. Snoeck, B. Belhadji, L. Calmels, F. Bertran, P. LeFevre, and A. TalebIbrahimi, Phys. Rev. B 82, 092405 (2010). 